\title{
Women's Knowledge About Indoor Air Pollution in Nepal
}

\section{Swikriti Acharya ${ }^{1,2}$, Madhu Koirala Dhital ${ }^{1,3 *}$, Kiran Sapkota ${ }^{4}$, Ganesh Shrivastav $^{5}$ and Shalik Ram Dhital ${ }^{6}$}

${ }^{1}$ National Open College, Sanepa, Kathmandu, Nepal

${ }^{2}$ Tribhuvan University, Manmohan Memorial Institute of Health Sciences, Kathmandu, Nepal

3 John Hunter Hospital, NSW, Newcastle, Australia

${ }^{4}$ University of Iowa, Iowa City, Iowa, USA

${ }^{5}$ Ministry of Health and Population, Kathmandu, Nepal

${ }^{6}$ National Health Education, Information and Communication Center, Kathmandu, Nepal

*Corresponding Author: Madhu Koirala Dhital, National Open College, Sanepa,

Kathmandu, Nepal.
Received: November 17, 2021

Published: January 21, 2022

(C) All rights are reserved by Madhu Koirala

Dhital., et al.

\section{Abstract}

Introduction: Indoor air pollution (IAP) is a major public health challenge in developing countries like Nepal. Annually, approximately 4.3 million people die due to IAP. In Nepal, $80 \%$ of rural households use biomass fuel for cooking without ventilation. This study to explore knowledge about IAP among women in the Dang district of Nepal.

Methods: A cross-sectional study was conducted in Tulsipur municipality of Nepal in 2016. Systematic random sampling was used, and data were collected using a structured questionnaire about IAP at their household level. The study population was among women living in a household. The descriptive analysis was done using SPSS software and statistical analysis carried out in frequencies and percentages and data are shown in table, pie chart and bar graph. Each study variable was labeled, coded, and measured systematically.

Results: The total study participants were 196 women aged 15 years and above. This study showed that approximately $90 \%$ of women had heard about IAP. Of these $28 \%$ and $24 \%$ of women said IAP is mainly caused due to cooking habits and poor ventilation respectively. Approximately 53\% of women were aware of ventilation and $46 \%$ agreed to have proper ventilation in the kitchen. Twenty-seven percentages of women agreed that health effects such as eye irritation was due to IAP where by headache $23 \%$, respiratory diseases $20 \%$ and so on.

Conclusion: Knowledge about IAP is a pragmatic way to prevent and mitigate the effects of IAP in the Dang, Nepal.

Keywords: Indoor Air Pollution; Knowledge; Rural; Women; Nepal

\section{Introduction}

Indoor air pollution (IAP) is often considering a neglected, public health problems. The common sources of IAP are biomass fuel such as wood, crop wastes, charcoal, coal, and dung for cooking and heating in the kitchen [1]. Biomass fuel is most common in developing countries such as Nepal. Globally, approximately 3 billion people burn biomass on open fires for daily cooking $[2,3]$. This produces heavy smoke containing carbon monoxide and polycyclic aromatic hydrocarbons [4]. 
The World Health Organization has estimated that 4.3 million people die because of households air pollution in 2018 [5]. Excessive exposure to IAP cause death due to pneumonia (27\%), stroke (18\%), ischemic heart disease (27\%), chronic obstructive pulmonary disease, and lung cancer (8\%) [6]. Women and children under five years are at high risk from IAP, especially in developing countries [3]. In Nepal, 66\% of households use solid fuel in 2016 [7]. Rural women and their children have a 2-3 times higher chance of respiratory disease including disability-adjusted life years [4,8]. A wide range of public health and energy consumption interventions can improve knowledge about IAP for women [9]. This study aims to explore knowledge about IAP among women in the Dang district of Nepal.

\section{Methods}

A cross-sectional study was carried out in Dang district of Nepal in 2016. A systematic random sampling technique was used for the selection of study population. A total of 196 women aged 15 years and above were selected for the survey. The women who were less than 18 were taken consent from her parents either grandmother or senior family member during the interview. Face to face interviews were conducted with women who were asked questionnaires related to IAP. Women were asked during the survey whether they were exposed to IAP or not. Structured questionnaires were used to collect data from the participants. A pilot study was carried out among women $(n=10)$ from the non-study area with similar background and necessary changes were made on the survey questionnaires. All questionnaires were rechecked just after completion of interview and questionnaire was made clear and simple. Women who were unable to respond due to their hearing impairment and dumbness were excluded from this study. The study protocol and thesis was approved by the institutional research Committee of National Open College, affiliated to the Pokhara University. Permission was sought from the Tulsipur municipality before data collection. Verbal informed consent was obtained from each participant. Participants' confidentiality was maintained. The outcome variable was in Likert scale. Participants received a score out of 100. Participants with a knowledge score below 25 scored were categorized as having poor knowledge, and higher than 25 scored were categorized as having good knowledge. In Statistical analysis it was dichotomously coded as Yes or No form. Age was measured as a continuous variable which was categorized and coded as: 15-24 = $1,25-34=2,35-44=3,45-54=4,55-64=5,65+=6$. The type of family was coded as nuclear $=1$ and Joint $=2$. Ethnicity was coded as Brahmin $=1$, Chhetri $=2$ Janjati $=3$. The source of income was categorized as agriculture $=1$, labour $=2$, business $=3$ and others $=4$. Education was categorized as Illiterate $=1$, Primary level $=2$, Secondary level $=3$, Higher secondary level, and above $=4$. Occupation was categorized in agriculture $=1$, labor $=2$, business $=3$, and other $=4$. Descriptive statistics were presented in frequency and percentage performed in SPSS. The level of knowledge was shown in the pie chart using MS Excel.

\section{Results}

Table 1 describes the demographic characteristics of women surveyed for knowledge on IAP. The highest representation of women were between 25-34 years 68(34.7\%). The most household was a nuclear family $112(57.1 \%)$, and approximately half of the participants were represented from Brahmin caste in this study. The major sources of incomes of the participants were agriculture 83(42\%), labour work 38(19\%), business 30(15\%) and non-specified other incomes $45(23 \%)$. Thirty-four percent of the women were illiterate, and $144(74 \%)$ had the main occupation of agriculture work.

In this study $177(90 \%)$ of women had heard about IAP and $19(10 \%)$ did not, Table 2 . The knowledge about the definition of IAP was varied among the women: wood burning 99(56\%), faulty gas $31(17 \%)$, nitrogen oxide $30(17 \%)$ and carbon monoxide $17(10 \%)$. Knowledge about the causes of IAP was cooking habits $81(46 \%)$, poor ventilation $69(39 \%)$, poverty $57(32 \%)$, illiteracy $61(35 \%)$, and other causes such as tobacco smoke and pesticides $18(10 \%)$. The table 2 also shows awareness of the types of solid fuel which cause IAP was 158(89\%) due to wood, 27(15.3\%) due to charcoal, $70(40 \%)$ due to dug, $113(64 \%)$ due to wastes and $39(22 \%)$ due to non-specified other causes. When women were asked about the type of stove, they had heard of $112(63 \%)$ know about gas, 69(39\%) know about kerosene stove, 118(67\%) know about mud tradition, and 99(56\%) know about improved stoves. The result has shown, about more than half of the participants 94(53\%) knew that there should be good ventilation with having a big hole in the kitchen, 48(27\%) with medium hole and 35(20\%) had poor ventilation with small hole in the kitchen.

Exposure to indoor air pollutants can lead to a wide range of adverse health outcomes such as Eye irritation 121(68\%), headache 
$100(57 \%)$, respiratory problems $91(52 \%)$, lung diseases 64(32\%), nausea $43(24 \%)$, and others like dry cough and fever $26(15 \%)$. The result also shows about knowledge of preventive measures of IAP among women were using alternative fuel 109(61.6\%), keeping proper ventilation $77(44 \%)$, dry ventilation $24(14 \%)$ and using improved stoves $53(30 \%)$ at household levels. The primary controlling mechanism of IAP were alternative fuels 99(56\%), window open $76(43 \%)$, proper ventilation $69(39 \%)$, and others control measures $16(9 \%)$.

\begin{tabular}{|c|c|c|}
\hline Characteristics & Options & Number(percentage) \\
\hline \multicolumn{3}{|c|}{ Age of women (year) } \\
\hline & $15-24$ & $17(8.7)$ \\
\hline & $25-34$ & $68(34.7)$ \\
\hline & $35-44$ & $47(24.0)$ \\
\hline & $45-54$ & $31(15.8)$ \\
\hline & $55-64$ & $20(10.2)$ \\
\hline & 65 and above & $13(6.6)$ \\
\hline \multicolumn{3}{|l|}{ Type of family } \\
\hline & Nuclear & $112(57.1)$ \\
\hline & Joint & $84(42.8)$ \\
\hline \multicolumn{3}{|l|}{ Ethnicity } \\
\hline & Brahmin & $96(49.0)$ \\
\hline & Chhetri & $32(16.3)$ \\
\hline & Janjati & $68(34.7)$ \\
\hline \multicolumn{3}{|l|}{ Source of income } \\
\hline & Agriculture & $83(42.4)$ \\
\hline & Labour work & $38(19.4)$ \\
\hline & Business & $30(15.3)$ \\
\hline & Non-specified & $45(23.0)$ \\
\hline \multicolumn{3}{|l|}{ Education } \\
\hline & Illiteracy & $66(33.7)$ \\
\hline & Primary & $42(21.4)$ \\
\hline & Secondary & $57(29.1)$ \\
\hline & Higher and above & $31(15.8)$ \\
\hline \multicolumn{3}{|l|}{ Occupation } \\
\hline & Agriculture & $144(73.5)$ \\
\hline & Labor & $6(3.1)$ \\
\hline & Business & $17(8.7)$ \\
\hline & Non-specified & $29(14.8)$ \\
\hline
\end{tabular}

Table 1: Demographic Characteristics of surveyed women

$$
\text { ( } \mathrm{n}=196) \text {. }
$$

Figure 1 shows approximately $\mathrm{n}=147(74.8 \%)$ of women have got more than 25 scores, and they were categorized as good knowledge and only $n=49(25.2 \%)$ of them have poor knowledge about the IAP.

\begin{tabular}{|c|c|c|}
\hline Variables & Options & Number (Percentage) \\
\hline \multicolumn{3}{|c|}{ Heard about IAP } \\
\hline & Yes & $177(90.3)$ \\
\hline & No & 19(9.7) \\
\hline \multicolumn{3}{|c|}{ IAP definitions } \\
\hline & Wood burning & $99(55.9)$ \\
\hline & Faulty gas & $31(17.5)$ \\
\hline & Nitrogen oxide & $30(17.0)$ \\
\hline & Carbon Monoxide & $17(9.6)$ \\
\hline \multicolumn{3}{|c|}{ Causes of IAP } \\
\hline & Cooking habit & $81(45.8)$ \\
\hline & Poor ventilation & $69(39)$ \\
\hline & Poverty & $57(32.2)$ \\
\hline & Illiteracy & $61(34.5)$ \\
\hline & Non-specified & $18(10.2)$ \\
\hline \multicolumn{3}{|c|}{ Type of solid fuel } \\
\hline & Wood & $158(89.3)$ \\
\hline & Charcoal & $27(15.3)$ \\
\hline & Dug & $70(39.5)$ \\
\hline & Waste & $113(63.8)$ \\
\hline & Non-specified & $39(22.0)$ \\
\hline \multicolumn{3}{|c|}{ Type of stoves } \\
\hline & Gas & $112(63.3)$ \\
\hline & Kerosene & $69(39.0)$ \\
\hline & Mud tradition & $118(66.7)$ \\
\hline & Improve stoves & $99(55.9)$ \\
\hline \multicolumn{3}{|c|}{ Size of Ventilation } \\
\hline & Small & $39(19.8)$ \\
\hline & Medium & $53(27.1)$ \\
\hline & Big & $104(53.1)$ \\
\hline \multicolumn{3}{|c|}{ Health Hazards } \\
\hline & Respiratory diseases & $91(51.7)$ \\
\hline & Lungs diseases & $64(36.2)$ \\
\hline & Eye irritation & $121(68.4)$ \\
\hline & Nausea & $43(24.3)$ \\
\hline & Headache & $100(56.5)$ \\
\hline & Non-specified & $26(4.7)$ \\
\hline \multicolumn{3}{|c|}{ Prevention of IAP } \\
\hline & Alternative fuels & $109(61.6)$ \\
\hline & Proper ventilation & $77(43.5)$ \\
\hline & Dry ventilation & $24(13.6)$ \\
\hline & Improved stoves & $53(29.9)$ \\
\hline \multicolumn{3}{|c|}{ Control of IAP } \\
\hline & Window open & $76(42.9)$ \\
\hline & Alternative fuels & $99(55.9)$ \\
\hline & Proper ventilation & $69(39.0)$ \\
\hline & Non-specified & $16(9.0)$ \\
\hline
\end{tabular}

Table 2: Knowledge of women related to Indoor Air Pollution ( $=177)$. 


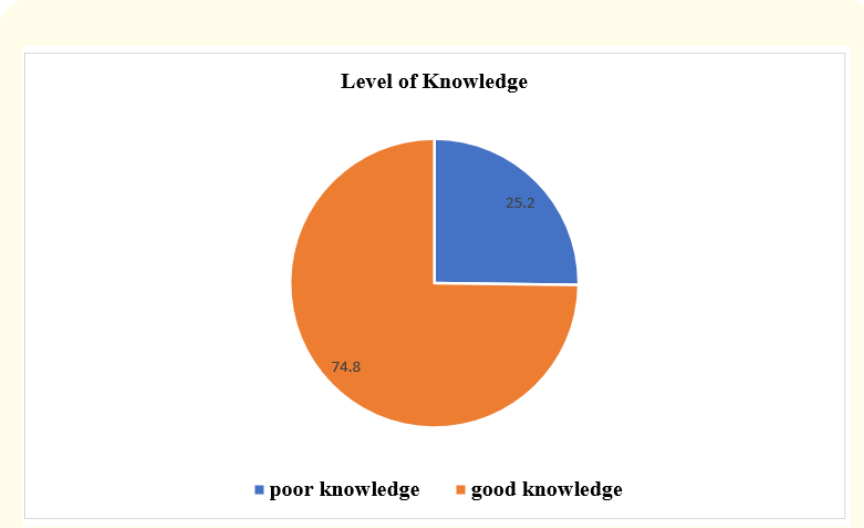

Figure 1: Women's knowledge about Indoor Air Pollution $(\mathrm{N}=177)$.

\section{Discussion}

The aim of this study was to explore knowledge about IAP among women in the Dang district of Nepal. This study related to knowledge about IAP variables included women heard about IAP, definition of IAP, causes of IAP, types of solid fuel, types of stoves, size of stove and ventilation, health hazards due to IAP, and prevention and control measures of IAP.

This study showed that one third of participants had good knowledge about IAP. The majority of participants had understanding that the main source of IAP was burning wood. The women expressed about the health hazards due to IAP were respiratory infection, lungs diseases, eyes irritation, nausea, and headache.

Approximately $20 \%$ of the study women understood the cause of IAP was due to poor ventilation which was four times lower than a previous study conducted in Pakistan in 2018 [10]. Approximately $90 \%$ of the participants were known of some methods to reduce IAP exposure in Rural Pakistan among women rather than the vast majority of more than nine in ten were aware of the availability of clean fuel such as LPG, and they have expressed willingness to switch it. However, more than half of the participants still use biomass fuel, and only a few participants were using a combination of biomass fuel and LPG [11]. This may be due to a lack of knowledge and poor economic status.

Unhealthy ventilated kitchen, rooms along with dampness and absence of daylight, were the main factors contributing to the diseases comprising 56\% [4]. A study conducted in Pakistan, mentioned that the common source of fuels were natural gas, LPG and a combination of fuels. When considering the cooking environment, participants were aware of strategies such as improving ventilation, use of a chimney and better kitchen design. In modifying the user behaviour by changing the cooking practice, the use of dry fuel was proposed by all the participants who declared awareness [11]. Ventilation plays an important role in indoor air improvement [12].
For the knowledge about the IAP in the household, it is very important to know about indoor air quality, their health impact as well as prevention and control measure of IAP to the women engage in the kitchen. Exposure to IAP can lead to a wide range of adverse health outcomes showed in this study which was similar with previous studies carried out in Kathmandu [13]. Moderate to severe cases of ARI was also reported in early age children $(<2 \mathrm{yr})$ because of domestic smoke exposure [14]. Due to excessive smoking and exposure to the burning of biomass fuels, multiple diseases, such as chronic bronchitis and emphysema, were reported in males, and indoor smoking is an important risk factor in terms of disease incidence and mortality burdens $[13,15]$. Similarly, approximately three quarter participants reported that the IAP cause cough [10]. Most of the people were aware that cooking fuels have health impacts and 8 in 10 knew that some fuels were better for health than others [11]. The participants' knowledge of the health effect of exposure to IAP was higher of the participants had poor knowledge of the health effects of prolonged exposure to air pollution from indoor cooking, while about one in ten participants had good knowledge of the health effects due to prolonged exposure to IAP [16]. Knowledge regarding the cause of ARI was seen in participants were about four in five and agreed IAP cause ill-health was more than four in five in the related study [17]. The latest study showed that women's education level did not have any significant difference of stunting due to IAP among children under five years in one of the hill districts Salyan of Nepal [18]. This similarity was because of the Salyan and Dang district loos almost the similar with education, culture, and household cooking habits.

The household socio-economics status determined fuel choice and clean indoor practices [19]. Most of the women spent their time indoors, which resulted in chronic exposure to indoor allergens and irritants. As the result of our study, only half of the participants have shown knowledge of preventive measures of IAP among women. Similarly, in a similar study, those who knew about IAP was detrimental to health were $95 \%$, and there was an association between IAP and respiratory diseases [10]. This is due to the lack of awareness of the harmful effects of polluting indoor air.

The study provides important baseline information about the level of knowledge of women about the indoor air pollution. This study will be useful in developing tailored education programs regarding causes, health consequences, prevention and control. Policymakers may feel a strong need to address IAP as a public health and health promotion issue if they had specific information about the economic burden from the direct and indirect costs of IAP. Besides, messages might need to be targeted to the household family members including household women for the effective prevention and control strategies. Additional research can be gathered through this information would be warranted and planned as a follow-up to this study. 
Knowledge of IAP remains one of the most pragmatic ways that can work effectively in preventing and mitigating the effects of IAP. Campaigns in rural areas by both the government and the local media and the health systems (for example Primary Health Care Out Reach Clinic, Health Mother Group, Health facilities etc.) run by health workers can be utilized to increase the level of knowledge and awareness campaign would be provided to control IAP. Capacity building of the community members may help to improve their economic wellbeing, thereby improving access to alternative cooking methods. Therefore, the strategies and policy should be introduced to promote alternative fuels, improve cook stoves, and different mass media campaigns. There is a need for massive and widespread health promotion campaigns to raise awareness among the people to increase the level of knowledge on the acute and chronic health risks of IAP. Besides, the findings suggest that public health programs need to intervene to increase the level of knowledge about IAP and to reduce its health hazards due to IAP. A personal strategy is also required to mitigate air pollution [20]. Urgent integrated health education services and energy consumption strategies are needed to improve the status of cooking fuel use at the household level in Nepal [21]. The context-specific strategies and plans of IAP and health promotion at the district and provincial level must be established in Nepal.

The strength of the study is sample of the diversity ethnic groups and socio-economic and ethnic groups represented. Unlike previous studies based on focused settings or ethnic groups. This study was carried out in the poor and rural communities within a municipality which can give real picture of knowledge level about IAP. However, this study may not be presented without limitations. This study was cross-sectional and cannot make causal inference. Further, this study have low sample size which requires further study with a large sample. As such, the sample may not fully represent the actual situation of the women population in the areas of Nepal. The study also does not included the smoking as IAP throughout the survey, which can be one of the great issues. The study only concerns the level of knowledge of IAP, but further analytical and interventional studies can be conducted to establish the causal association.

\section{Conclusions}

This study concluded that surveyed women had good knowledge about indoor air pollution was almost three times higher than poor knowledge in this study. Very few women were aware of Liquefied petroleum gas, and natural gas is better ways of reducing pollution. However the importance of proper ventilation in the kitchen and the size of the kitchen were known by interviewed women.

\section{Acknowledgement}

We express our gratitude to study participants and acknowledged to Tulsipur municipality staff. We also would like to thanks to research and development department (an Institutional research review committee) at National Open College (NOC), Sanepa Kathmandu, Nepal for approving study protocol and thesis. We thank to Mr Maheshor Kaphle, Public Health Coordinator and Principal Dr Sital Kumar Karki from the NOC for their support and guidance. We also would like to thank Mr Samip Dhital from the University of Newcastle, Australia for the final review of language.

\section{Conflict of Interest}

None

\section{Authors' Contribution}

All authors contributed equally. The concept, design, planning and data analysis (MKD and SA); Ethical application and thesis conducted (SA); data cross verification and reference management (SRD); Literature review (GS and SRD); Draft manuscript (MKD, SA and SRD); Manuscript revision all authors; Final manuscript: all authors; Accountability of the work: all authors.

\section{References}

1. Bruce N., et al. "IAP in developing countries: a major environmental and public health challenge". Bulletin of the World Health Organization 78 (2000): 1078-1092.

2. Sumpter C and Chandramohan D. "Systematic review and meta-analysis of the associations between IAP and tuberculosis". Tropical Medicine and International Health 18.1 (2013): 101-108.

3. Lee KK., et al. "Adverse health effects associated with household air pollution: a systematic review, meta-analysis, and burden estimation study". The Lancet Global Health 8.11 (2020): e1427-e1434.

4. Thapa B and Chaurasia N. "IAP due to Inadequate Ventilation and its Impact on Health among Children of Less Than Five Years in Eastern Nepal". Journal of Nobel Medical College 3.1 (2014): 35-39. 
5. World Health Organization. "Household air pollution and health" (2018).

6. Apte JM. "A study of relationship between background characteristics, media exposure and acceptance of family planning in rural Maharashtra". IIPS News 29.4 (1988): 13-22.

7. Ministry of Health and Population. Nepal Demographic and Health Survey. Kathmandu: Ministry of Health and Population, New ERA and Inner City Fund International (2016).

8. Joshi H., et al. "IAP and its Health Impact on People of Malikarjun Village Development Community, Darchula district (A case study)". Nepal Health Research Council (2008).

9. Von Schirnding Y., et al. "Addressing the Impact of Household Energy and IAP on the Health of Poor: Implications for Policy Action and Intervention Measures". World Health Organization Geneva (2002).

10. Tariq N., et al. "Awareness about IAP in general population of Rawalpindi and Islamabad". Pakistan Journal of Public Health 8.2 (2018): 80-83.

11. Nasir ZA., et al. "Ethno-environmental knowledge as a tool to combat IAP in low income countries: A case study from rural communities in Pakistan" (2014).

12. Zhao Y., et al. "Indoor air environment of residential buildings in Dalian, China". Energy and Buildings 36.12 (2004): 12351239.

13. Shrestha IL and Shrestha SL. "IAP from biomass fuels and respiratory health of the exposed population in Nepalese households". International Journal of Occupational and Environmental Health 11.2 (2005): 150-160.

14. Pandey M., et al. "Domestic smoke pollution and acute respiratory infections in a rural community of the hill region of $\mathrm{Ne}$ pal”. Environment International 15.1-6 (1989): 337-340.

15. Junaid M., et al. "Status of IAP (IAP) through particulate matter (PM) emissions and associated health concerns in South Asia". Chemosphere 191 (2018): 651-663.

16. Isara AR and Aigbokhaode AQ. "Household cooking fuel use among residents of a sub-urban community in Nigeria: implications for IAP". The Eurasian Journal of Medicine 46.3 (2014): 203.

17. Myat KKS., et al. "IAP: Impact of intervention on acute respiratory infection (ARI) in under-five children". Regional Health Forum (2005).
18. Koirala M., et al. "Effects of IAP on Stunting Among Children Aged 1-5 Years in Salyan District of Nepal”. Acta Scientific Paedriatrics 4.3 (2021): 10-16.

19. Ghimire S., et al. "Geographic and socio-economic variation in markers of IAP in Nepal: evidence from nationally-representative data". BMC Public Health 19.1 (2019): 1-5.

20. Carlsten C., et al. "Personal strategies to minimise effects of air pollution on respiratory health: advice for providers, patients and the public". European Respiratory Journal 55.6 (2020).

21. Shupler M., et al. "Household and personal air pollution exposure measurements from 120 communities in eight countries: results from the PURE-AIR study". The Lancet Planetary Health 4.10 (2020): e451-462.

\section{Assets from publication with us}

- Prompt Acknowledgement after receiving the article

- Thorough Double blinded peer review

- Rapid Publication

- Issue of Publication Certificate

- High visibility of your Published work

Website: www.actascientific.com/

Submit Article: www.actascientific.com/submission.php

Email us: editor@actascientific.com

Contact us: +919182824667 